\title{
Synthesis of pyranopyrazolo $N$-glycoside and pyrazolopyranopyrimidine $C$-glycoside derivatives as promising antitumor and antimicrobial agents
}

\author{
HEND N. HAFEZ ${ }^{1,2}$ \\ ABDEL-RHMAN B. A. EL-GAZZAR ${ }^{1,2}$ \\ ${ }^{1}$ Al-Imam Mohammad Ibn Saud \\ Islamic University (IMSIU) \\ Faculty of Science \\ Department of Chemistry \\ P.O. Box 90950 Riyadh, 11623 \\ Kingdom of Saudi Arabia \\ ${ }^{2}$ Photochemistry Department \\ Heterocyclic and Nucleosides Unit \\ National Research Centre \\ Cairo, Egypt
}

\begin{abstract}
As a part of systematic investigation of the synthesis and biological activities of pyrazole analogues linked to various heterocyclic systems, a new series of pyrazolo- $N$-glycoside derivatives, pyrazolopyranopyrimidine and $C$-glycoside of pyrazolopyranotriazolo-pyrimidine derivatives was synthesized through the reaction of the key intermediate 6-amino-3-methyl-4-(substituted-phenyl)-1,4-dihydropyrano[2,3c]pyrazole-5-carbonitrile $(\mathbf{3} \mathbf{a}, \mathbf{b})$ with different reagents. Structures of the newly synthesized compounds were elucidated by elemental microanalysis and spectroscopic methods. The compounds were subjected to in vitro antitumor evaluation using the MTT assay. $N-(\beta$ - $D$-ribofuranosyl)- and $N$-( $\beta$-D-xylofuranosyl)-6\{[(1E)-4-chlorophenyl)-methylene] amino\}4-(4-florophenyl)-3-methyl-1,4-dihydropyrano[2,3-c]pyrazole-5-carbonitrile $(\mathbf{6} \mathbf{a}, \mathbf{b})$ were the most active compounds against three human cancer cell lines. Also, most of the newly synthesized compounds exhibited high activity towards Gram-negative and Gram-positive bacteria. Compound 6a exhibited excellent activity towards bacteria compared to ofloxacine as the reference drug.
\end{abstract}

Keywords: $N$-nucleoside of pyranopyrazole, C-glycoside of pyrazolopyranotriazolopyrimidine, anticancer activity, antimicrobial activity

Nucleoside analogs play an important role in anticancer chemotherapy (1). Cancer cells display a strong ability of acquiring resistance to antitumor drugs, termed multidrug resistance (MDR), which is a critical hurdle to cancer therapy. For this reason, the discovery and development of new types of highly selective anticancer agents is still a very urgent topic. Pyrazolone nucleus has attracted much attention due to its interesting biological activities (2-5). The chemistry of pyrazole derivatives received great attention because of their biological activities as potential HIV-1 inhibitors (6), insecticides, fungicides (7), antiviral agents (8) and anticancer activity (9). On the other hand, fused pyrazoles such as

\footnotetext{
*Correspondence; e-mail: profelgazzar@yahoo.com; abgazzar@imamu.edn.sa
} 
pyranopyrazoles are an important class of biologically active heterocycles. They have been reported to possess a multiplicity of pharmacological properties, including anticancer (10), antimicrobial (11), antiinflammatory (12), insecticidal and molluscicidal activities $(13,14)$. They are also potential inhibitors of human Chk1 kinase (15) and can find applications as pharmaceutical ingredients and biodegradable agrochemicals (16).

In the course of our ongoing research on modified heterocyclic and nucleoside analogs, we became interested in a stereo-selective strategy for the preparation of $N$-nucleosides. Moreover, the increasing biological importance of pyranopyrazole derivatives, particularly in chemotherapy, prompted us to develop and identify new molecules, such as pyrano[2,3-c]-pyrazolo-carbonitrile derivatives $3 \mathbf{a}, \mathbf{b}$, the corresponding pyrazolo $\left[4^{\prime}, 3^{\prime}: 5,6\right]$ pyrano[2,3- $d]$-pyrimidines $\mathbf{1 0 a , b}$ and $C$-glycoside derivatives $\mathbf{1 2} \mathbf{a}-\mathbf{d}$ and $\mathbf{1 4 a} \mathbf{a}-\mathbf{d}$, as well as pyrano[2,3- $d]$-pyrazolo-carbonitrile derivatives $\mathbf{4} \mathbf{a}, \mathbf{b}$ and the corresponding $N$-glycosides 6a-f and 10a-d, in order to investigate the effect of structural modifications on their antimicrobial and antitumor activities.

\section{EXPERIMENTAL}

All melting points were measured on an Electrothermal 9100 series digital melting point apparatus (Shimadzu, Japan). Microanalytical data were obtained with a Vario Elementar apparatus (Shimadzu). Elemental analyses of all compounds were within $\pm 0.4 \%$ of the theoretical values. Physicochemical data are given in Table I. The IR spectra (KBr) were recorded on a Perkin Elmer 1650 spectrometer (USA). ${ }^{1} \mathrm{H}$ NMR spectra were recorded on a JEOL EX-300 and a JEOL ECA-500 (Japan). Chemical shifts were expressed in ppm relative to $\mathrm{SiMe}_{4}$ as internal standard and DMSO- $d_{6}$ as solvent. Mass spectra were recorded on a 70 ev EI Ms-QP 1000 EX (Shimadzu). Spectral data are given in Tables II and III. Thin layer chromatography (chloroform/methanol, 8:2) indicated the formation of pure compounds. Compounds ethyl-3-(2-carbamothioyl-hydrazinylidene) butanoate (1) and arylidene malononitriles $(\mathbf{2} \mathbf{a}, \mathbf{b})$ were obtained using the procedures reported elsewhere $(17,18)$. Ethyl acetoacetate, thiosemicarbazide, 1-bromo-2,3,5-tri-O-acetyl- $\alpha$ - $D$-ribo-/or xylo and arabinofuranose, 2,3,4,6-tetra- $O$-acetyl- $\alpha$ - $D$-gluco- and galacto pyranosyl bromide were purchased from Sigma-Aldrich (USA). Ofloxacine, miconazole and doxorubicin disks were supplied by the Pasteur Laboratory (Giza, Egypt).

\section{Syntheses}

6-Amino-4-aryl-3-methyl-1,4-dihydropyrano[2,3-c]pyrazole-5-carbonitriles $(3 \boldsymbol{a}, \boldsymbol{b})$. General procedure. - Ethyl-3-(2-carbamo-thioylhydrazineylidene)-butanoate (3) (2.03 g, $10 \mathrm{mmol})$ and then arylidene malononitrile $(\mathbf{2} \mathbf{a}, \mathbf{b})(10 \mathrm{mmol})$ were added into a sodium ethoxide solution $(20 \mathrm{~mL})$. The reaction mixture was heated under reflux for $6 \mathrm{~h}$. The product obtained was crystallized from an appropriate solvent to produce $\mathbf{3} \mathbf{a}, \mathbf{b}$, respectively, in high yields.

6-(4-Chloro-phenylmethylenehydrazone)-4-substituted phenyl-3-methyl-1,4-dihydropyrano[2,3-clpyrazole-5-carbonitriles $(\mathbf{4} \boldsymbol{a}, \boldsymbol{b})$. General procedure. - The compounds were obtained from $\mathbf{3 a}, \mathbf{b}$ and $p$-chlorobenzaldehyde in ethanol/piperidine. The reaction mixture was heated 


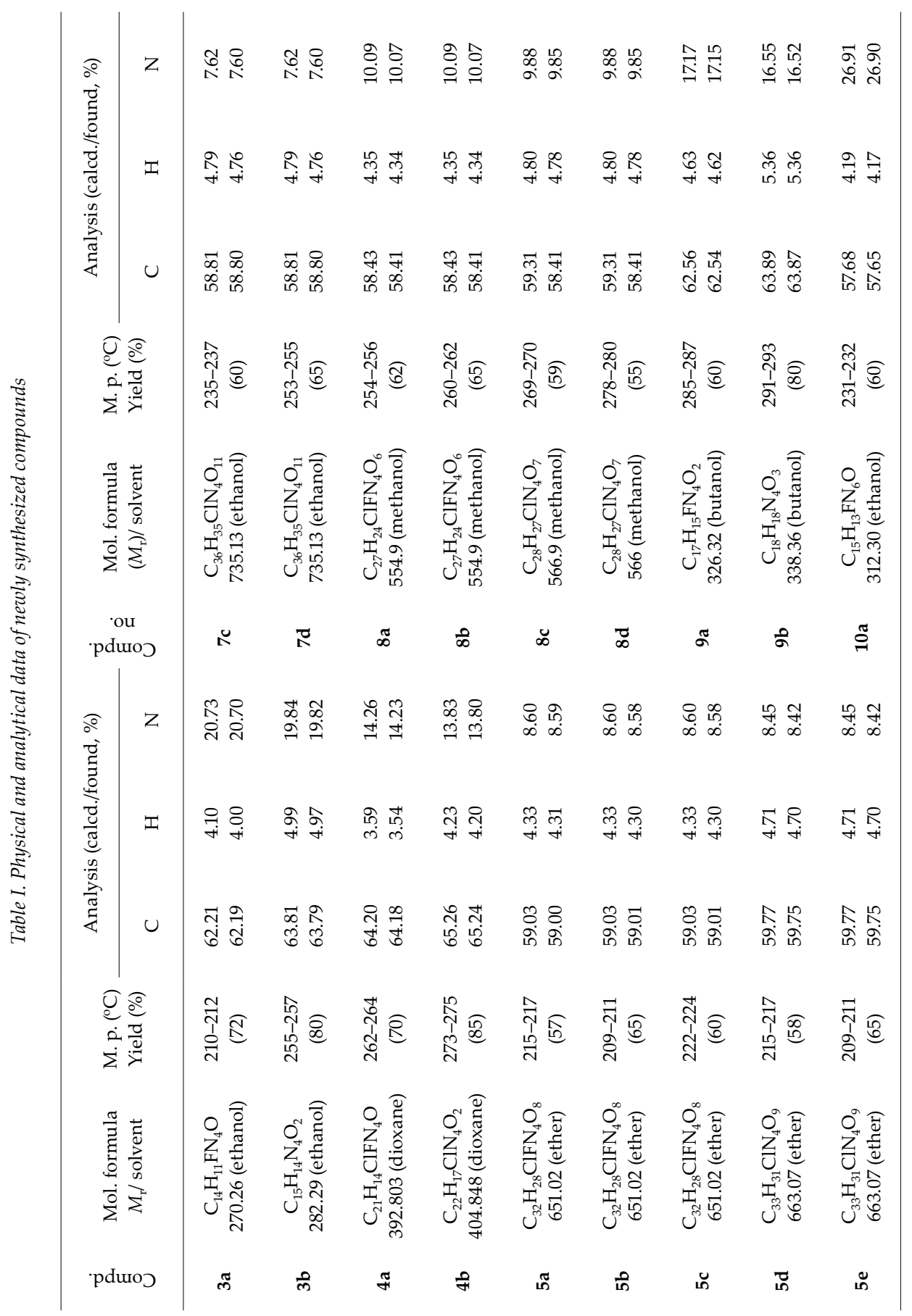




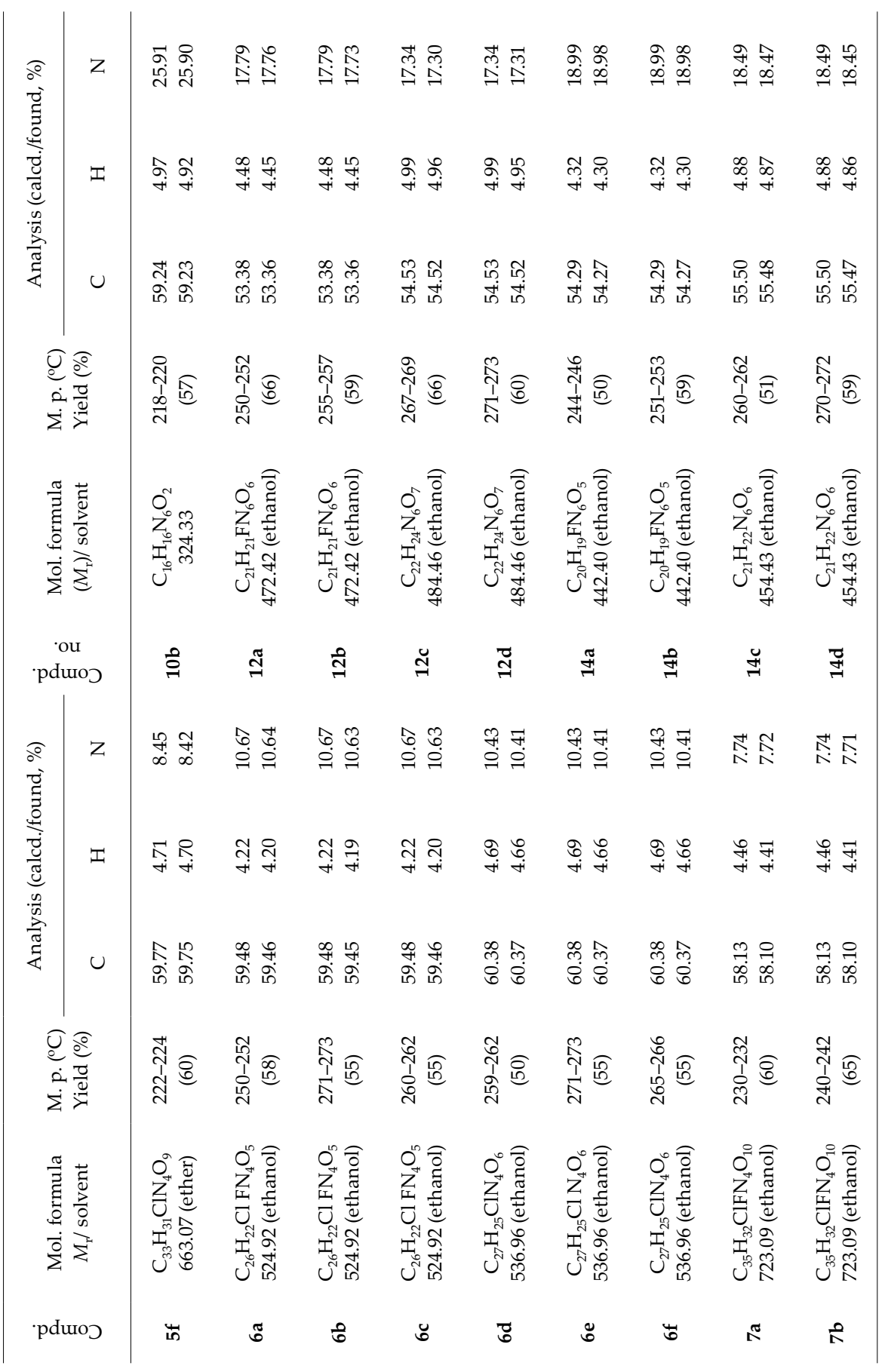


under reflux for $8 \mathrm{~h}$. The product obtained was crystallized from an appropriate solvent to produce $\mathbf{4 a}, \mathbf{b}$ in good yields.

Acetylated N-nucleosides of 6\{[(1E)-4-chlorophenyl)methylene]amino\}4-substituted phenyl-3methyl-1,4-dihydro-pyrano[2,3-c]pyrazole-5-carbonitriles (5a-f and $7 \boldsymbol{a}$-d). General procedure. - To a solution of $4 \mathbf{a}, \mathbf{b}(0.01 \mathrm{~mol})$ in $5 \mathrm{~mL}$ of aqueous potassium hydroxide $(0.01 \mathrm{~mol})$, a solution of 1-bromo-2,3,5-tri-O-acetyl- $\alpha$ - $D$-ribo-/or xylo and arabinofuranose or 2,3,4,6-tetra-Oacetyl- $\alpha$ - $D$-gluco-/or galacto and mannosopyranosyl bromide $(0.015 \mathrm{~mol})$ in acetone (40 $\mathrm{mL}$ ) was added. The reaction mixture was stirred at room temperature for $24 \mathrm{~h}$ (under TLC control). The solvent was evaporated under reduced pressure at $40^{\circ} \mathrm{C}$, the crude product was filtered off and washed with distilled water to remove the $\mathrm{KBr}$ formed. The product was dried and crystallized from the proper solvent to produce $\mathbf{5 a - f}$ and $\mathbf{7 a - f}$, respectively.

Deacetylated N-( $\beta$-D-furanosyl and glycosyl) 6\{[(1E)-4-chlorophenyl) methylene]amino\}4-(4floro-/4-methoxyphenyl)-3-methyl-1,4-dihydropyrano[2,3-c]pyrazole-5-carbonitriles (6a-f or $8 \boldsymbol{a}$ d). General procedure. - Dry gaseous ammonia was passed through a solution of acetylated compounds 5 a-f or $7 \mathbf{a}-\mathbf{d}(1.0 \mathrm{mmol})$ in dry methanol $(20 \mathrm{~mL})$ at room temperature for 10 min. The mixture was stirred overnight. The resulting mixture was then evaporated under reduced pressure to afford a solid residue, which was crystallized from appropriate solvent to afford $\mathbf{6 a}-\mathbf{f}$ and $\mathbf{8 a}-\mathbf{d}$, respectively.

Ethyl [5-cyano-4-(4-substituted-phenyl)-3-methyl-1,4-dihydropyrano[2,3-c]pyrazol-6-yl] imido-formates $(\mathbf{9} \boldsymbol{a}, \boldsymbol{b})$. General procedure. - To a mixture of triethyl orthoformate $(0.01 \mathrm{~mol})$ and acetic anhydride $(20 \mathrm{~mL})$, compounds $5 \mathbf{a}, \mathbf{b}(0.01 \mathrm{~mol})$ were added and the reaction mixture was refluxed for $5 \mathrm{~h}$. The solvent was removed under reduced pressure and the separated solid was recrystallized from appropriate solvent to give $\mathbf{9 a}, \mathbf{b}$.

4-(4-Substituted-phenyl)-5-imino-3-methyl-1,4-dihydropyrazolo[4',3':5,6]pyrano[2,3-d]pyrimidin-6(5H)-amines $(\mathbf{1 0} \boldsymbol{a}, \boldsymbol{b})$. General procedure. - To a solution of $\mathbf{9 a}, \mathbf{b}(0.01 \mathrm{~mol})$ in absolute ethanol $(50 \mathrm{~mL})$, hydrazine hydrate $(0.01 \mathrm{~mol})$ was added. The reaction mixture was refluxed for $2 \mathrm{~h}$, concentrated, cooled, and the solid product that separated out was filtered off and recrystallized from appropriate solvent to give $\mathbf{1 0 a , b}$.

2-(Penta-O-acetyl/tetra-O-acetyl-glycosyl)-11-(4-florophenyl)-10-methyl-8,11-dihydropyrazolo-[4',3':5,6]pyrano[3,2-e][1,2,4]triazolo[1,5-c]pyrimidines (11a-d and 13a-d). - A solution from each of $10 \mathbf{a}, \mathbf{b}(10 \mathrm{mmol})$ and aldopentose/aldohexose $(10 \mathrm{mmol})$ in a mixture of acetic anhydride-acetic acid (1:1) (50 mL) was stirred under reflux for 3-5 h. The mixture was then extracted several times with chloroform (150-200 mL). After removal of chloroform under reduced pressure, the residue (intermediates 11a-d, 13a-d) was processed in the next step without identification.

2-(Glycosyl)-11-(4-florophenyl)-10-methyl-8,11-dihydropyrazolo[4',3':5,6]pyrano[3,2e]-[1,2,4]triazolo[1,5-c]pyrimidines (12a-d and 14a-d). General procedure. - A solution from each of 11a-d or 13a-d (10 mmol) in a solution of sodium methoxide (10 mmol) (sodium metal in methanol, $100 \mathrm{~mL}$ ) was stirred at room temperature for $24 \mathrm{~h}$ and then neutralized with hydrochloric acid solution ( $\mathrm{pH}$ control). The precipitate formed was filtered off, washed with cold water, dried and crystallized from ethanol (60-100 mL) to obtain 12a-d, and 14a-d, respectively. 


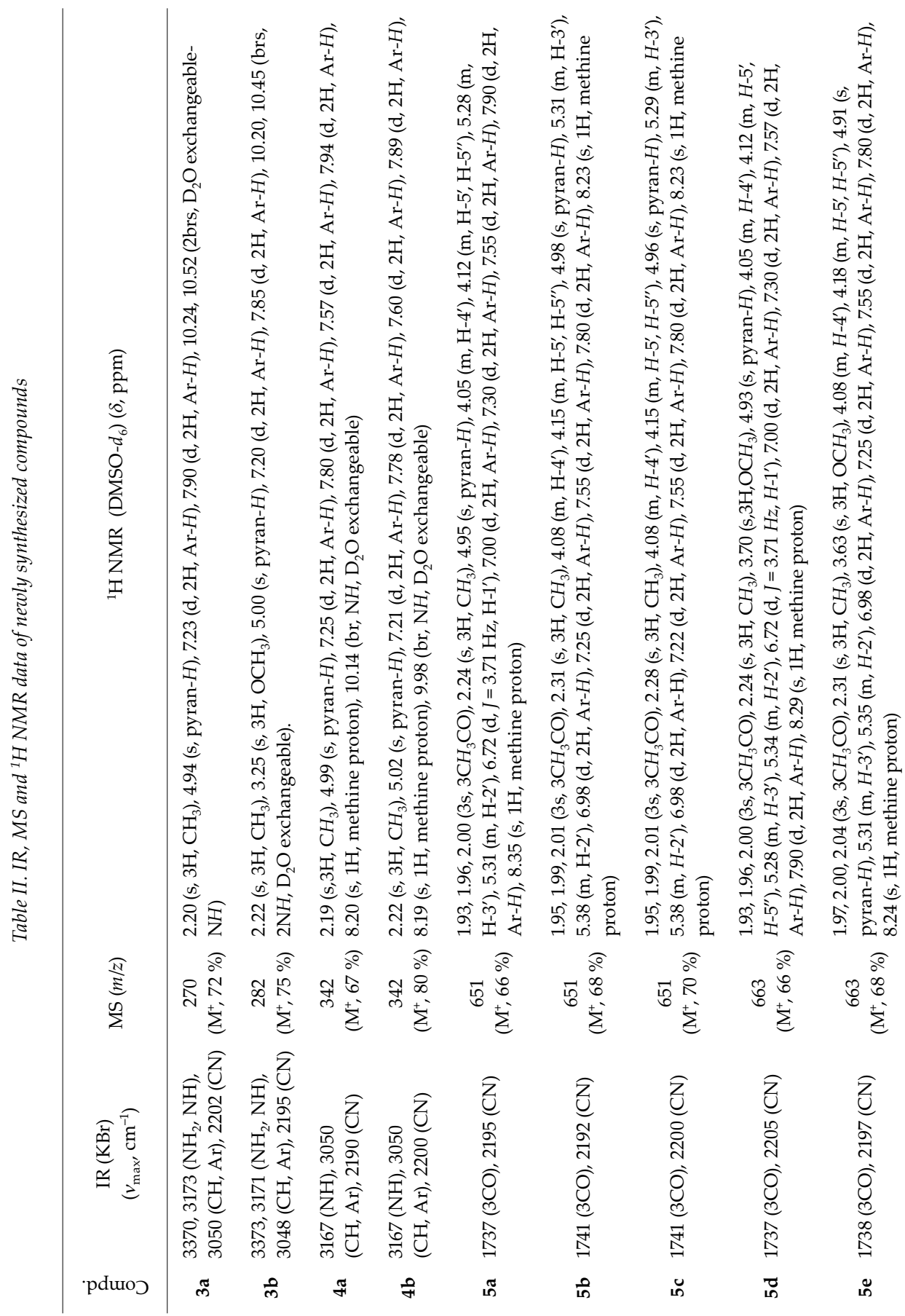




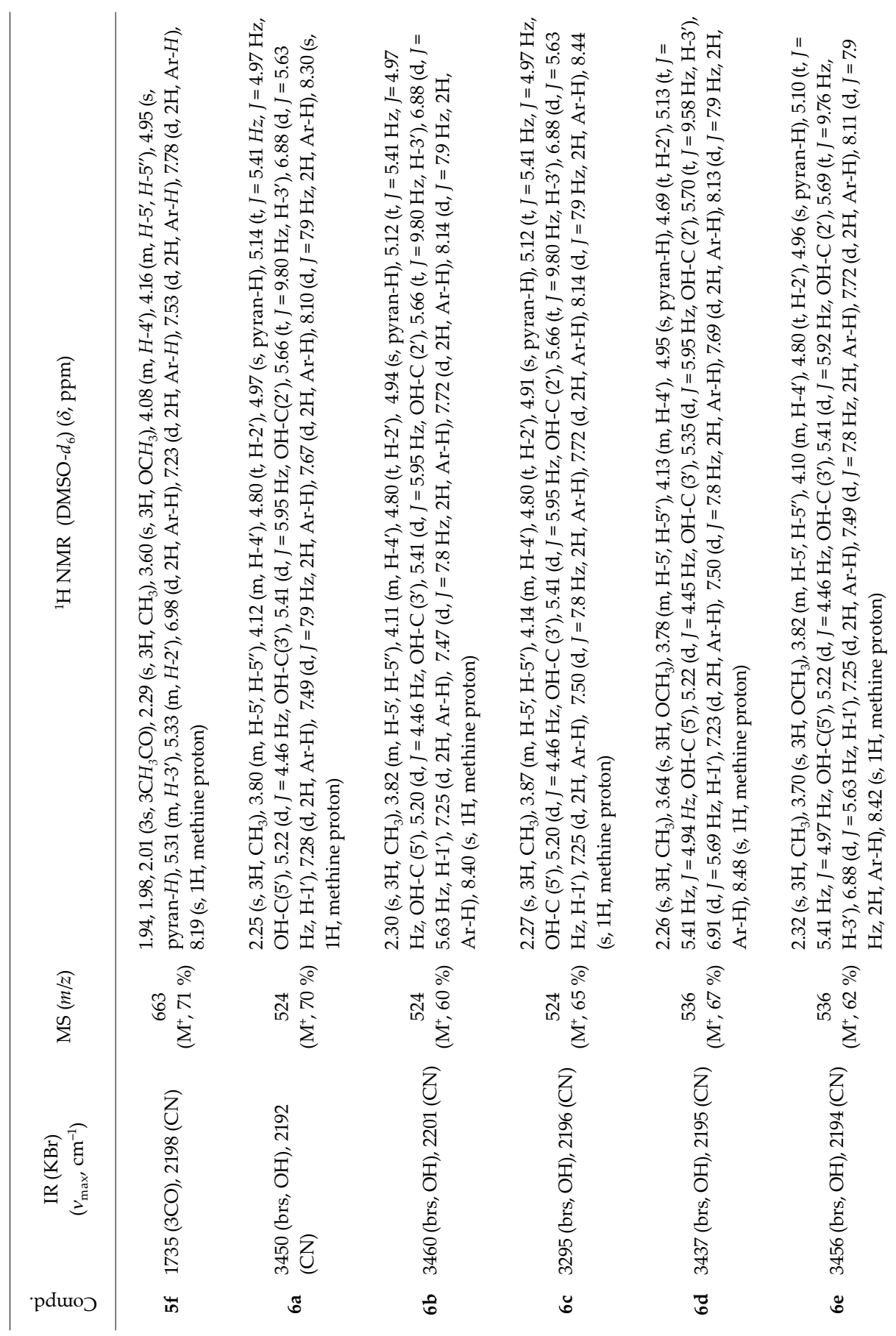




\begin{tabular}{|c|c|c|c|c|c|c|c|}
\hline 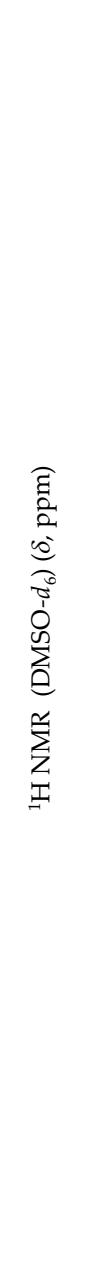 & 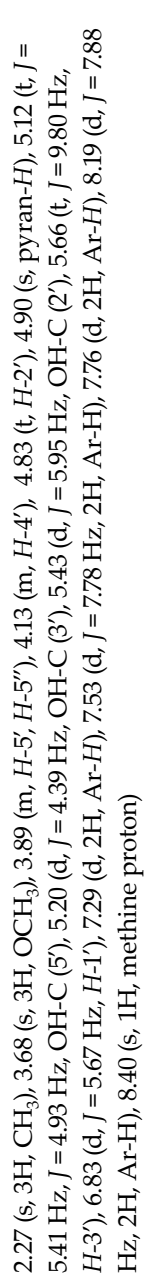 & 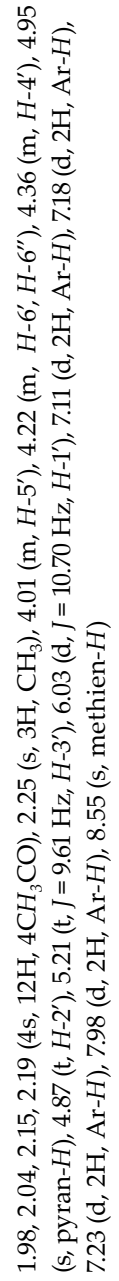 & 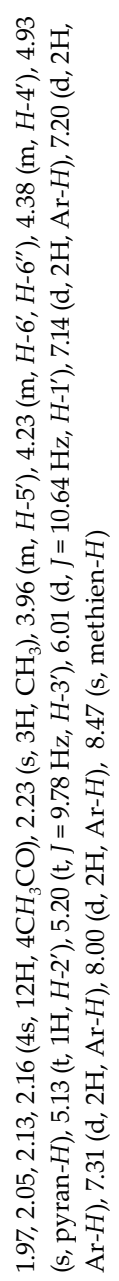 & 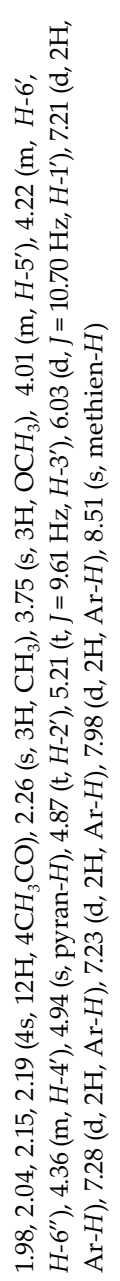 & 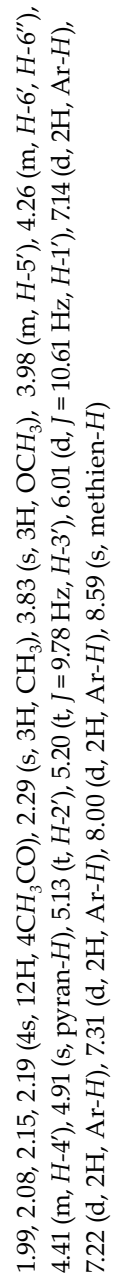 & 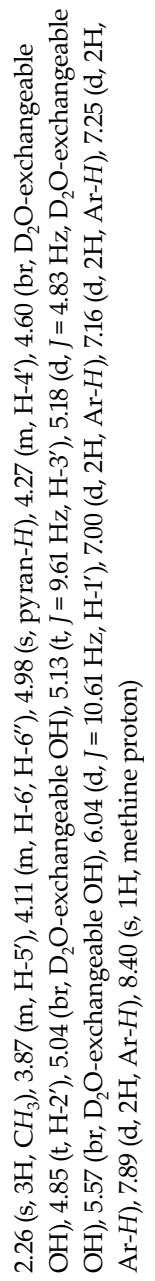 & 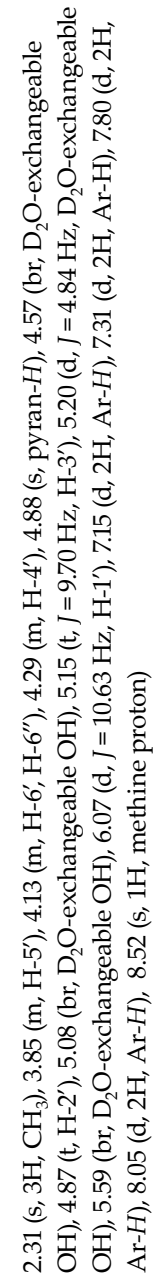 \\
\hline $\begin{array}{l}\frac{\pi}{\Sigma} \\
\sum \\
\sum\end{array}$ & 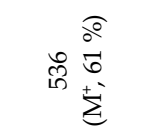 & 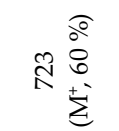 & 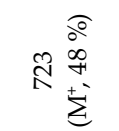 & 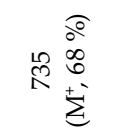 & 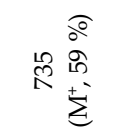 & 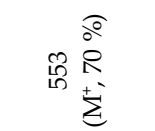 & 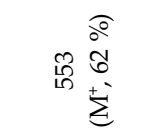 \\
\hline 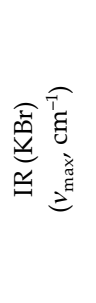 & 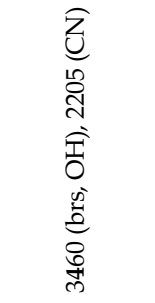 & 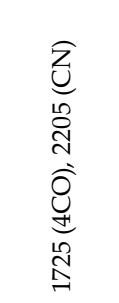 & 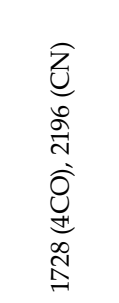 & 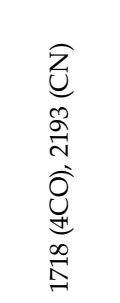 & 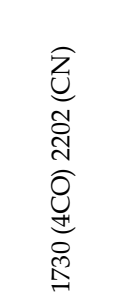 & 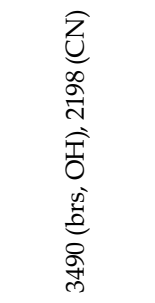 & 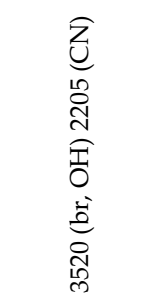 \\
\hline pduroכ & 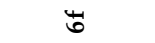 & ๙ & 2 & 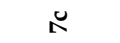 & $\pi$ & $\infty$ & $\infty$ \\
\hline
\end{tabular}




\begin{tabular}{|c|c|c|c|c|c|c|c|c|}
\hline 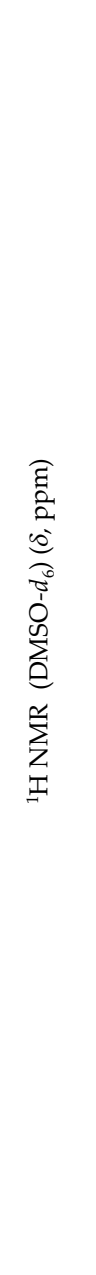 & 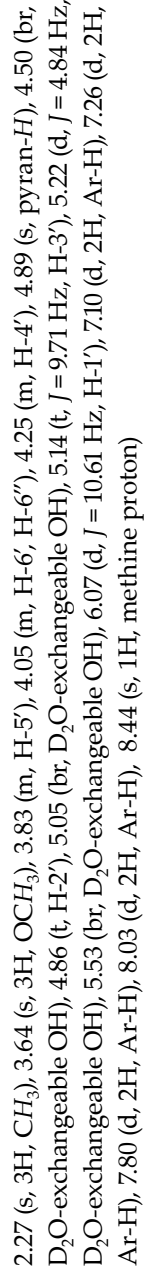 & 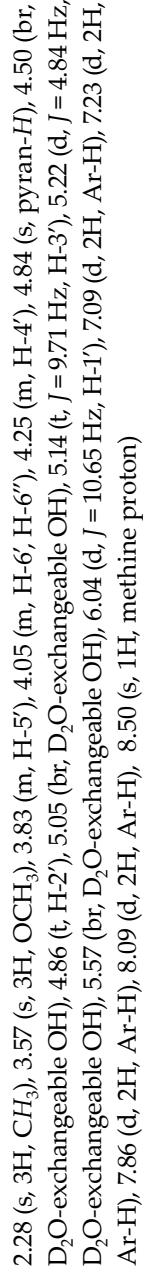 & 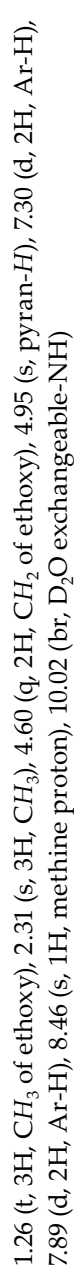 & 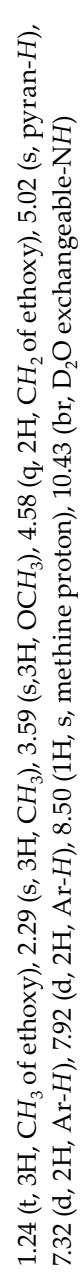 & 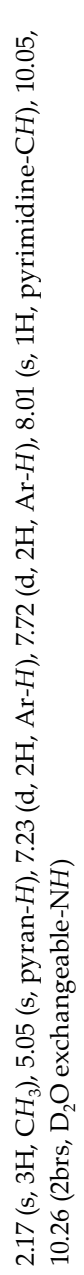 & 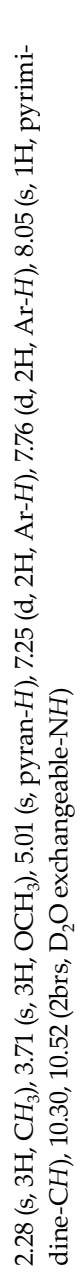 & 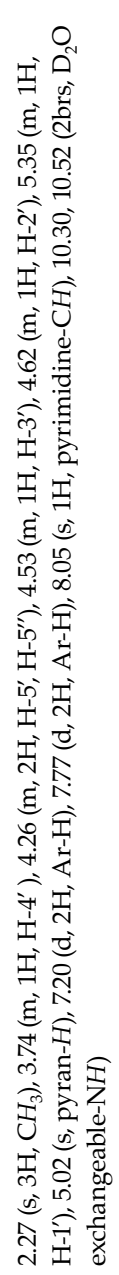 & 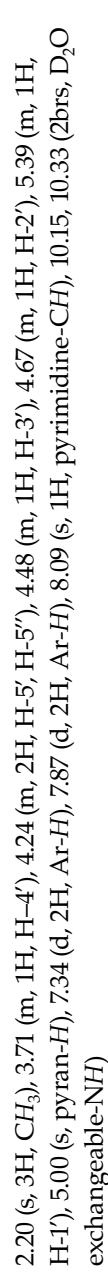 \\
\hline $\begin{array}{l}\frac{\pi}{\Sigma} \\
\equiv \\
\text { W }\end{array}$ & 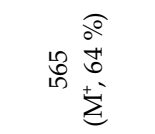 & 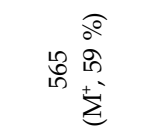 & 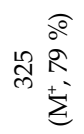 & 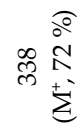 & 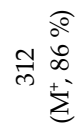 & 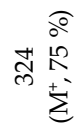 & 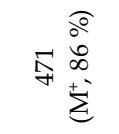 & $\begin{array}{l}2 \\
0 \\
\infty \\
\infty \\
\vdots \\
\sum\end{array}$ \\
\hline 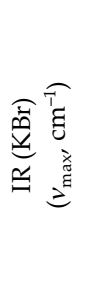 & 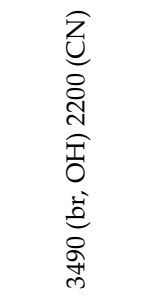 & 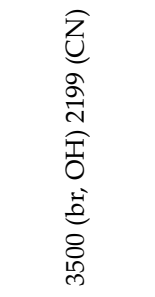 & 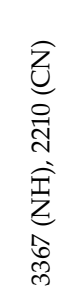 & 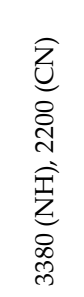 & 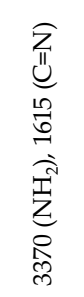 & 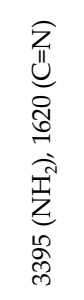 & 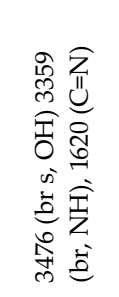 & 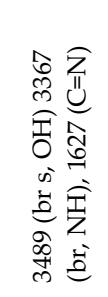 \\
\hline pduros & $\check{\infty}$ & రా & శ็ & ह & $\stackrel{\widetilde{\Omega}}{0}$ & 음 & త̃ & $\theta$ \\
\hline
\end{tabular}




\begin{tabular}{|c|c|c|c|c|c|c|}
\hline 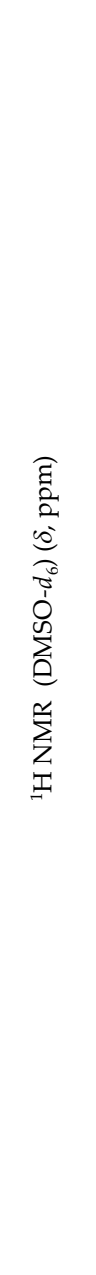 & 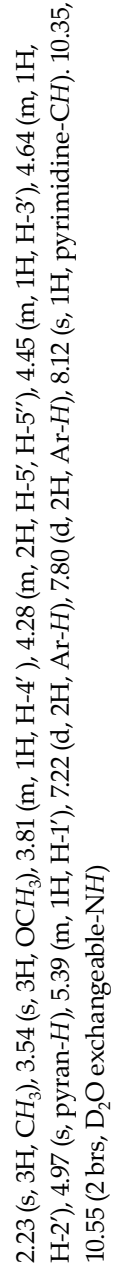 & 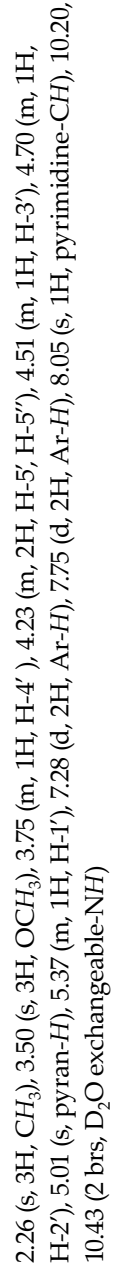 & 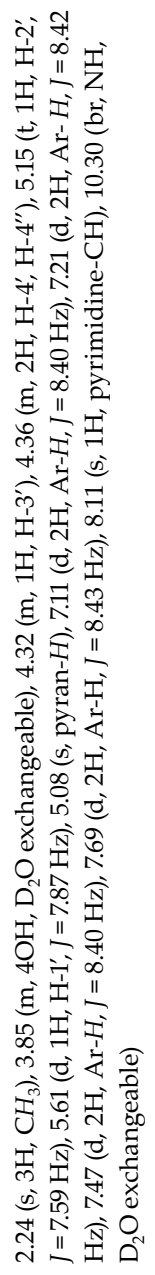 & 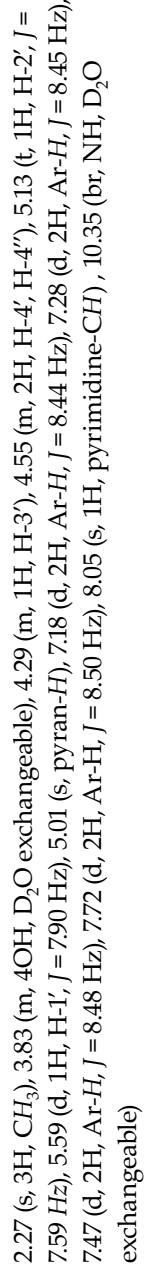 & 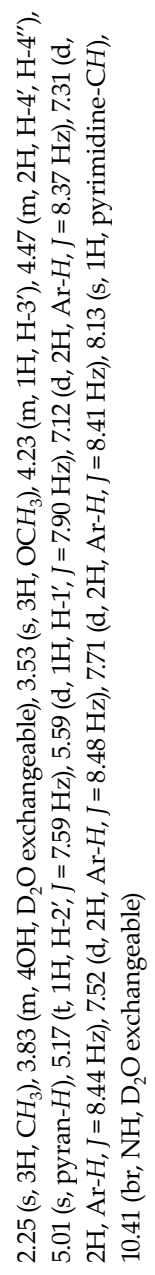 & 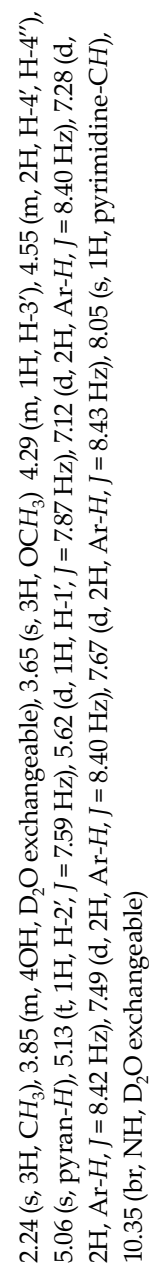 \\
\hline $\begin{array}{l}\frac{\pi}{\Xi} \\
\frac{\omega}{\Sigma}\end{array}$ & 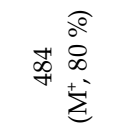 & 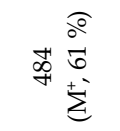 & 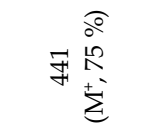 & 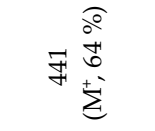 & 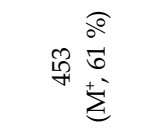 & 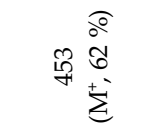 \\
\hline 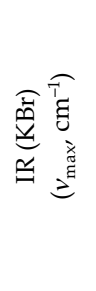 & 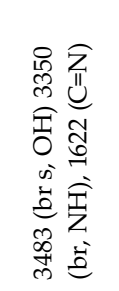 & 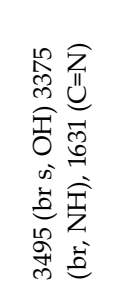 & 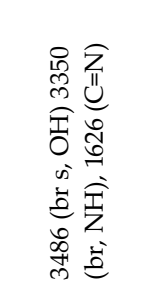 & 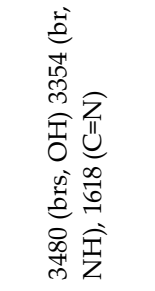 & 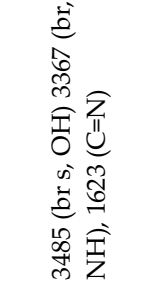 & 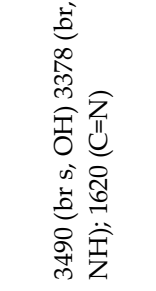 \\
\hline pduoJ & $\breve{I}$ & $\widetilde{\mathbb{I}}$ & Iี & F & I & I \\
\hline
\end{tabular}


H. N. Hafez and A-R. B. A. El-Gazzar: Synthesis of pyranopyrazolo N-glycoside and pyrazolopyranopyrimidine C-glycoside derivatives as promising antitumor and antimicrobial agents, Acta Pharm. 65 (2015) 215-233.

Table III. ${ }^{13} \mathrm{C}$ NMR data of newly synthesized compounds

\begin{tabular}{|c|c|}
\hline Compd. & ${ }^{13} \mathrm{C}$ NMR $\left(\mathrm{DMSO}-d_{6}\right)(\delta, \mathrm{ppm})$ \\
\hline $6 a$ & $\begin{array}{l}19.91\left(\mathrm{CH}_{3}\right), 60.86\left(\mathrm{C}-5^{\prime}\right), 65.34\left(\mathrm{C}-3^{\prime}\right), 67.54\left(\mathrm{C}-2^{\prime}\right), 69.35\left(\mathrm{C}-4^{\prime}\right), 87.43\left(\mathrm{C}-\mathrm{l}^{\prime}\right), 89.30(\mathrm{C}-4 \\
\text { pyran}), 119.1(\mathrm{CN}), 125.6-158.5(18 \mathrm{C} \text {, two phenyl and pyranopyrazole rings) }\end{array}$ \\
\hline $6 b$ & $\begin{array}{l}20.08\left(\mathrm{CH}_{3}\right), 60.79\left(\mathrm{C}-5^{\prime}\right), 65.40\left(\mathrm{C}-3^{\prime}\right), 67.55\left(\mathrm{C}-2^{\prime}\right) 69.42\left(\mathrm{C}-4^{\prime}\right), 87.51\left(\mathrm{C}-\mathrm{l}^{\prime}\right), 88.56(\mathrm{C}-4 \text { pyran}), \\
118.3(\mathrm{CN}), 126.2-157.9(18 \mathrm{C} \text {, phenyl and pyranopyrazole rings) }\end{array}$ \\
\hline $8 a$ & $\begin{array}{l}20.09\left(\mathrm{CH}_{3}\right), 61.49\left(\mathrm{C}-6^{\prime}\right), 66.76\left(\mathrm{C}-3^{\prime}\right), 68.41\left(\mathrm{C}-2^{\prime}\right), 68.98\left(\mathrm{C}-4^{\prime}\right), 77.83\left(\mathrm{C}-5^{\prime}\right), 88.86\left(\mathrm{C}-\mathrm{l}^{\prime}\right), 89.57 \\
(\mathrm{C}-4 \text { pyran}), 117.9(\mathrm{CN}), 124.8-158.7(18 \mathrm{C} \text {, two phenyl and pyranopyrazole rings) }\end{array}$ \\
\hline $8 b$ & $\begin{array}{l}20.11\left(\mathrm{CH}_{3}\right), 61.38\left(\mathrm{C}-6^{\prime}\right), 66.72\left(\mathrm{C}-3^{\prime}\right), 68.37\left(\mathrm{C}-2^{\prime}\right), 68.95\left(\mathrm{C}-4^{\prime}\right), 77.61\left(\mathrm{C}-5^{\prime}\right), 88.91\left(\mathrm{C}-\mathrm{l}^{\prime}\right), 90.08 \\
(\mathrm{C}-4 \text { pyran}), 118.5(\mathrm{CN}), 123.9-157.8(18 \mathrm{C} \text {, two phenyl and pyranopyrazole rings) }\end{array}$ \\
\hline $12 a$ & $\begin{array}{l}21.28\left(\mathrm{CH}_{3}\right), 30.83\left(\mathrm{CH}_{2}\right), 67.70,68.45,69.24 \text { and } 70.52(4 \mathrm{CH}), 89.67(\mathrm{C}-4 \text { pyran}), 126.4-155.6 \\
(14 \mathrm{C} \text {, phenyl and pyrazolopyrano-triazolo-pyrimidine rings) }\end{array}$ \\
\hline $12 b$ & $\begin{array}{l}20.58\left(\mathrm{CH}_{3}\right), 31.28\left(\mathrm{CH}_{2}\right), 67.81,68.50,69.33 \text { and } 71.42(4 \mathrm{CH}), 90.25(\mathrm{C}-4 \text { pyran}), 125.6-154.7 \\
(14 \mathrm{C} \text {, phenyl and pyrazolopyrano-triazolo-pyrimidine rings) }\end{array}$ \\
\hline $14 a$ & $\begin{array}{l}21.38\left(\mathrm{CH}_{3}\right), 29.89\left(\mathrm{CH}_{2}\right), 67.83,69.45 \text { and } 71.59(3 \mathrm{CH}), 89.53(\mathrm{C}-4 \text { pyran}), 121.4-153.9(14 \mathrm{C} \text {, } \\
\text { phenyl and pyrazolopyrano-triazolo-pyrimidine rings) }\end{array}$ \\
\hline $14 b$ & $\begin{array}{l}21.38\left(\mathrm{CH}_{3}\right), 29.89\left(\mathrm{CH}_{2}\right), 67.83,69.45 \text { and } 71.59(3 \mathrm{CH}), 89.53(\mathrm{C}-4 \text { pyran}), 121.4-153.9(14 \mathrm{C} \text {, } \\
\text { phenyl and pyrazolopyrano-triazolo-pyrimidine rings) }\end{array}$ \\
\hline
\end{tabular}

\section{Pharmacological screening}

Antimicrobial activity. - Antimicrobial activities of the new synthesized compounds $\mathbf{4 a}, \mathbf{b}, \mathbf{6 a}, \mathbf{b}, \mathbf{d}, \mathbf{8 a}, \mathbf{b}, \mathrm{d}, \mathbf{1 0 a}, \mathbf{b}, \mathbf{1 2} \mathbf{a}, \mathbf{d}$ and $\mathbf{1 4 a}, \mathbf{b}, \mathbf{d}$ were tested in vitro against Gram-positive bacteria Bacillus subtilis NCTC-1040, Staphylococcus aureus NCTC-7547, Gram-negative bacteria Escherichia coli NCTC-10416, Sarcina lutea ATCC-954 and fungal strains Candida albicans ATCC- 14154 and Aspergillus flavus ATCC-23554. All microorganisms were purchased from the American Type Culture Collection (Manassas, VA, USA). The compounds were dissolved in DMSO (5 mg mL $\left.\mathrm{m}^{-1}\right)$ and tested for antimicrobial activity by the agar disk diffusion technique (19) using a 1-cm microplate-well diameter and a $100 \mu \mathrm{L}$ of each concentration. Compound impregnated disks were placed on an agar plate containing a standard suspension of microorganisms. The plate was incubated for $24 \mathrm{~h}$ at $35^{\circ} \mathrm{C}$. The diameters of the zones of inhibition were measured with calipers or automated scanners and were compared with those of the standards. Ofloxacine $\left(50 \mu \mathrm{gL}^{-1}\right)$ and miconazole $\left(50 \mu \mathrm{g} \mathrm{mL}^{-1}\right)$ were used as standards for antibacterial and antifungal activity, respectively. The observed zones of inhibition are shown in Table IV.

The minimal inhibitory concentration (MIC) of the test compounds was determined by the agar streak dilution method (20). Stocks of synthesized compounds were prepared by dissolving $1 \mathrm{mg}$ in $1 \mathrm{~mL}$ DMSO and diluted to the desired concentration. Different concentrations of the synthesized compounds were mixed with known quantities of mol- 
H. N. Hafez and A-R. B. A. El-Gazzar: Synthesis of pyranopyrazolo N-glycoside and pyrazolopyranopyrimidine C-glycoside derivatives as promising antitumor and antimicrobial agents, Acta Pharm. 65 (2015) 215-233.

Table IV. Inhibition zones for antibacterial and antifungal activities of the newly synthesized compounds

\begin{tabular}{ccccccc}
\hline & \multicolumn{5}{c}{ Inhibition zone diameter (mm) } \\
\cline { 2 - 7 } Compd. & \multicolumn{7}{c}{ Gram(+) bacteria } & Gram(-) bacteria & \multicolumn{2}{c}{ Fungi } \\
\cline { 2 - 7 } & B. subtilis & S. aureus & E. coli & S. lutea & C. albicans & A. flavus \\
\hline $\mathbf{4 a}$ & 18 & 17 & 19 & 16 & 7 & 8 \\
$\mathbf{4 b}$ & 17 & 18 & 16 & 19 & 9 & 10 \\
$\mathbf{6 a}$ & 25 & 23 & 26 & 25 & 8 & 9 \\
$\mathbf{6 b}$ & 23 & 21 & 23 & 23 & 8 & 7 \\
$\mathbf{6 d}$ & 21 & 21 & 23 & 23 & 8 & 6 \\
$\mathbf{8 a}$ & 22 & 21 & 23 & 23 & 12 & 13 \\
$\mathbf{8 b}$ & 21 & 20 & 22 & 22 & 8 & 7 \\
$\mathbf{8 d}$ & 23 & 19 & 21 & 21 & 8 & 9 \\
$\mathbf{1 0 a}$ & 18 & 17 & 15 & 18 & 7 & 8 \\
$\mathbf{1 0 b}$ & 15 & 16 & 17 & 16 & 6 & 7 \\
$\mathbf{1 2 a}$ & 19 & 20 & 21 & 20 & 16 & 18 \\
$\mathbf{1 2 d}$ & 17 & 19 & 18 & 18 & 11 & 11 \\
$\mathbf{1 4 a}$ & 20 & 21 & 21 & 21 & 9 & 8 \\
$\mathbf{1 4 b}$ & 19 & 21 & 21 & 21 & 9 & 8 \\
$\mathbf{1 4 d}$ & 19 & 19 & 20 & 21 & 9 & 8 \\
\hline Miconazole & - & - & - & - & 20 & 20 \\
\hline Ofloxacine & 25 & 25 & 25 & 25 & - & - \\
\hline
\end{tabular}

Inhibition zone: 6-10 $\mathrm{mm}$ - slight activity, 11-15 $\mathrm{mm}$ - moderate activity, > $15 \mathrm{~mm}$ - high activity

ten sterile agar medium. About $15 \mathrm{~mL}$ of the medium containing the test compound was poured into a sterile Petri dish and the medium was allowed to solidify. Microorganisms were then streaked one by one on the agar plates. After streaking, all the plates were incubated at $37^{\circ} \mathrm{C}$ for 24 and $48 \mathrm{~h}$ for antimicrobial and antifungal activity, respectively. The MIC values are shown in Table IV.

Antitumor activity. - Some of the synthesized compounds, namely, pyrazolo $N$-nucleoside $(\mathbf{6 a}, \mathbf{b}, \mathbf{d}, \mathbf{8 a}, \mathbf{d})$, and $C$-glycoside of triazolopyrimidine derivatives 12a and 14a were tested for in vitro anticancer activity against three human cancer cell lines [liver cancer (HepG2), colon cancer (HT29), breast adenocarcinoma (MCF-7)] by the MTT assay. The three human cancer cell lines were provided by the National Cancer Institute (NCI, Cairo, Egypt). Their growth as a monolayer was maintained in RPMI-1640 medium supplemented with $5 \%$ heat inactivated FBS (fetal bovine serum), $2 \mathrm{mmol} \mathrm{L}^{-1}$ glutamine and antibiotics (penicillin $100 \mathrm{U} \mathrm{mL}^{-1}$, streptomycin $100 \mu \mathrm{g} \mathrm{mL} \mathrm{L}^{-1}$ ), at $37^{\circ} \mathrm{C}$. Exponentially growing cells were obtained by plating $1.5 \times 10^{5}$ cells $\mathrm{mL}^{-1}$, followed by 24 -h incubation. The effect of the vehicle solvent DMSO on the growth of these cell lines was evaluated by exposing un- 
H. N. Hafez and A-R. B. A. El-Gazzar: Synthesis of pyranopyrazolo N-glycoside and pyrazolopyranopyrimidine C-glycoside derivatives as promising antitumor and antimicrobial agents, Acta Pharm. 65 (2015) 215-233.

Table V. MIC $\left(\mu \mathrm{mol} \mathrm{L} L^{-1}\right)$ of the newly synthesized compounds

\begin{tabular}{ccccccc}
\hline & \multicolumn{2}{c}{ Gram(+) bacteria } & \multicolumn{2}{c}{ Gram(-) bacteria } & \multicolumn{2}{c}{ Fungi } \\
\cline { 2 - 7 } Compd. & B. subtilis & S. aureus & E. coli & S. lutea & C. albicans & A. flavus \\
\hline $\mathbf{4 a}$ & 1.6 & 2.2 & 4.5 & 26.5 & 51.0 & 66.0 \\
$\mathbf{4 b}$ & 3.5 & 4.5 & 1.6 & 22.5 & 50.0 & 65.0 \\
$\mathbf{6 a}$ & 0.3 & 0.5 & 3.5 & 0.25 & 30.0 & 30.0 \\
$\mathbf{6 b}$ & 0.5 & 2.0 & 1.0 & 1.4 & 30.0 & 30.5 \\
$\mathbf{6 d}$ & 0.9 & 2.5 & 1.5 & 9.3 & 50.1 & 50.5 \\
$\mathbf{8 a}$ & 2.8 & 3.9 & 2.1 & 1.2 & $>100$ & 40 \\
$\mathbf{8 b}$ & 2.0 & 2.0 & 2.4 & 2.8 & 28.0 & 30.0 \\
$\mathbf{8 d}$ & 1.0 & 1.5 & 1.2 & 1.6 & 30.0 & 30.0 \\
$\mathbf{1 0 a}$ & 8.5 & 12.0 & 20.0 & 6.0 & 45.0 & 45.3 \\
$\mathbf{1 0 b}$ & 10.0 & 15.0 & 12.0 & 18.9 & $>100$ & 50.8 \\
$\mathbf{1 2 a}$ & 3.0 & 3.5 & 2.9 & 1.5 & 30.0 & 30.0 \\
$\mathbf{1 2 d}$ & 5.0 & 5.0 & 8.5 & 12.0 & 45.0 & 50.8 \\
$\mathbf{1 4 a}$ & 1.0 & 2.5 & 2.5 & 3.3 & 30.8 & 25.9 \\
$\mathbf{1 4 b}$ & 3.3 & 3.0 & 2.1 & 20.4 & 66.0 & $>100$ \\
$\mathbf{1 4 d}$ & 12.0 & 22.5 & 20.5 & 24.5 & 65.3 & 55.5 \\
\hline Miconazole & - & - & - & - & 1.4 & 5.0 \\
\hline Ofloxacine & 0.4 & 0.4 & 0.35 & 0.25 & - & - \\
\hline
\end{tabular}

Table VI. The effect of synthesized compounds on the growth of human liver cancer (HepG2), colon cancer (HT29), breast adenocarcinoma (MCF-7) tumor cell lines

\begin{tabular}{cccc}
\hline \multirow{2}{*}{ Compd. } & \multicolumn{3}{c}{$G_{50}\left(\mu \mathrm{mol} \mathrm{L}^{-1}\right)$} \\
\cline { 2 - 4 } & $\mathrm{HePG}_{2}$ & $\mathrm{HT} 29$ & MCF-7 \\
\hline $\mathbf{6 a}$ & $5.8 \pm 0.6$ & $3.9 \pm 0.8$ & $6.2 \pm 0.2$ \\
$\mathbf{6 b}$ & $6.7 \pm 0.2$ & $4.5 \pm 0.8$ & $6.8 \pm 0.9$ \\
$\mathbf{6 d}$ & $14.5 \pm 0.8$ & $11.9 \pm 0.6$ & $16.7 \pm 1.6$ \\
$\mathbf{8 a}$ & $11.9 \pm 0.6$ & $20.3 \pm 0.5$ & $15.2 \pm 1.1$ \\
$\mathbf{8 d}$ & $20.6 \pm 0.4$ & $22.3 \pm 1.5$ & $24.1 \pm 0.8$ \\
$\mathbf{1 2 a}$ & $25.6 \pm 0.7$ & $20.5 \pm 1.1$ & $23.2 \pm 4.8$ \\
$\mathbf{1 4 a}$ & $18.6 \pm 0.9$ & $24.3 \pm 0.8$ & $20.3 \pm 0.6$ \\
\hline Doxorubicin & $0.05 \pm 0.007$ & $0.09 \pm 0.008$ & $0.05 \pm 0.008$ \\
\hline
\end{tabular}

Results are given as concentrations that were able to cause $50 \%$ cell growth inhibition $\left(G I_{50}\right)$ after continuous exposure for $48 \mathrm{~h}$.

Mean \pm SEM of three independent experiments performed in duplicate. 
treated control cells to the maximum concentration ( $0.5 \%)$ of DMSO used in each assay. The effect of compounds on in vitro growth of human tumor cell lines was evaluated according to the procedure adopted by the National Cancer Institute (NCI, USA) using sulforhodamine B as protein binding dye to assess cell growth (21). Cells growing exponentially in 96-well plates were then exposed for $48 \mathrm{~h}$ to five serial concentrations of each compound, starting from a maximum concentration of $150 \mu \mathrm{g} \mathrm{mL}^{-1}$. After this exposure period, adherent cells were fixed, washed and stained. The bound stain was solubilized and the absorbance was measured and the growth inhibition of $50 \%\left(G I_{50}\right)$ was calculated (22). Doxorubicin was used as a reference compound (Table VI).

\section{RESULTS AND DISCUSSION}

\section{Chemistry}

The first step of our strategy was condensation of thiosemicarbazide (1) with ethyl acetoacetate 2 in ethanol, in the presence of triethylamine, to afford ethyl-3-(2-carbmothioyl-hydrazinylidene) butanoate (1), which upon treatment with arylidene malononitriles 2a,b gave 6-amino-4-(4-floro- and/or 4-methoxyphenyl)-3-methyl-1,4dihydropyrano[2,3-c]pyrazole-5-carbonitrile derivatives $3 \mathbf{a}, \mathbf{b}$ in good yields, via Michael addition followed by cyclization through nucleophilic attack. On the other hand, the treatment of compounds $3 \mathbf{a}, \mathbf{b}$ with aldehyde, namely, 4 -chlorobenzaldehyde or anisaldehyde, afforded $4 \mathbf{a}, \mathbf{b}$ (Scheme 1).

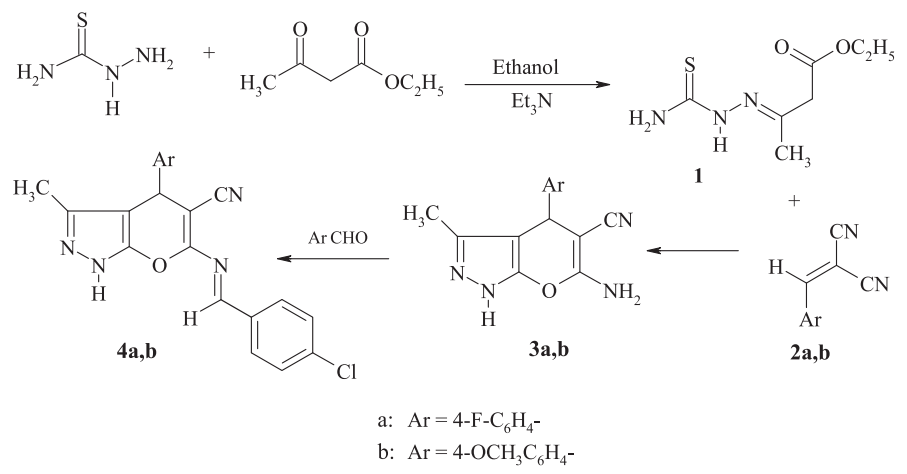

Scheme 1

Moreover, glycosylation of $\mathbf{4 a ,} \mathbf{b}$ with 2,3,5-tri- $O$-acetyl- $\beta$ - $D$-(ribo/xylo or arabino furanosyl) bromide or 2,3,4,6-tetra- $O$-acetyl- $\alpha$ - $D$-(glycol /galactosyl or mannosyl) bromide in acetone, in the presence of aqueous potassium hydroxide, afforded the corresponding acetylated nucleosides 5a-f and 7a-d, respectively, in good yields (57-65\%) (Schemes 2 and 3). Structures of $\mathbf{5 a - f}$ and $7 \mathbf{a}-\mathbf{d}$ were confirmed by elemental analyses and spectral data (IR, ${ }^{1} \mathrm{H}$ NMR). 


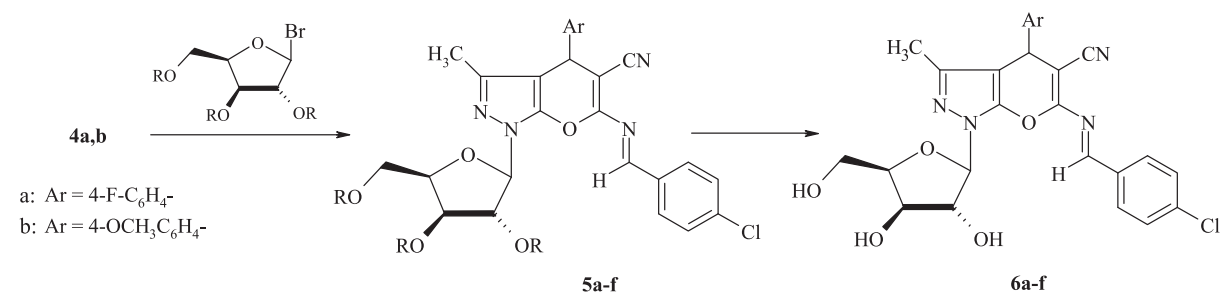

Scheme 2

As an example, ${ }^{1} \mathrm{H}$ NMR spectrum of compound 7a showed the anomeric proton of the glucose moiety as a doublet at $\delta 6.03 \mathrm{ppm}$ with a coupling constant $J=10.70 \mathrm{~Hz}$, indicating $\beta$-configuration of the anomeric center. Other protons of the glucopyranose ring resonated at $\delta 4.01-5.21 \mathrm{ppm}$, while the four acetoxy groups appeared as four singlet signals at $\delta$ 1.98, 2.04, 2.15, 2.19 ppm. Deprotection of acyclic nucleosides 5a-f and 7a-d could be achieved when they were stirred in a methanolic ammonia solution at room temperature, affording the corresponding deacetylated nucleosides 6a-f and 8a-d, respectively (Schemes 2 and 3). Structures of free nucleosides $\mathbf{6 a - f}$ and $\mathbf{8 a - d}$ were established on the basis of their spectral data and elemental analyses. The ${ }^{1} \mathrm{H}$ NMR spectrum of compound 6a showed the anomeric proton as a doublet at $\delta 6.88 \mathrm{ppm}$.
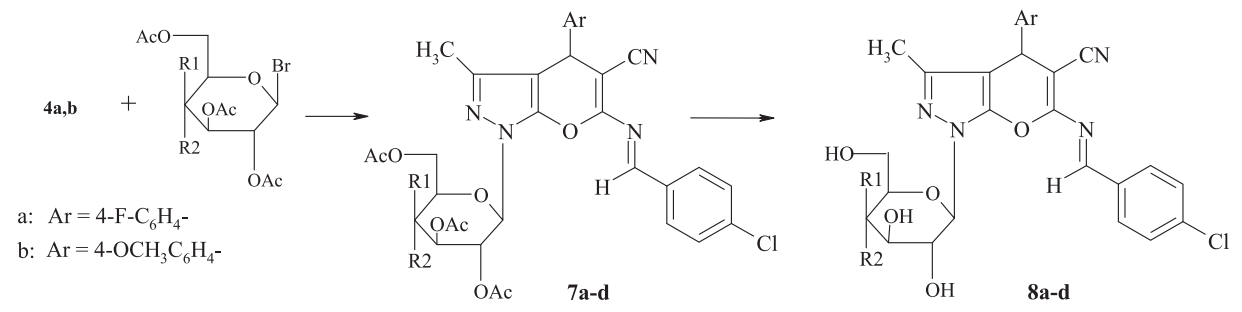

Scheme 3

Heating of $3 \mathbf{a}, \mathbf{b}$ with triethylorthoformate in boiling acetic anhydride gave the pyrano[2,3-c]pyrazole imidoformate derivatives $\mathbf{9 a}, \mathbf{b}$, which underwent cyclization, after the treatment with hydrazine hydrate to afford three fused rings, namely, 4-(4-substitutedphenyl)-5-imino-3-methyl-1,4-dihydro pyrazolo[4',3':5,6]pyrano[2,3-d]pyrimidin-6(5H)amines $\mathbf{1 0} \mathbf{a}, \mathbf{b}$. Heating of $\mathbf{1 0} \mathbf{a}, \mathbf{b}$ under reflux with stirring, with aldohexoses, namely, $D$ glucose and $D$-galactose, in a mixture of acetic anhydride-acetic acid (1:1) (23) afforded the intermediates 11a-d (Scheme 4).

Also, we extended our work to react compounds $10 \mathbf{a}, \mathbf{b}$ with aldopentoses, namely, $D$-ribose and $D$-arabinose. Thus, gentle heating of $\mathbf{1 0 a}, \mathbf{b}$ with stirring in a mixture of acetic anhydride-acetic acid yielded the corresponding intermediates $\mathbf{1 5 a - d}$, respectively, as shown in Scheme 5. 


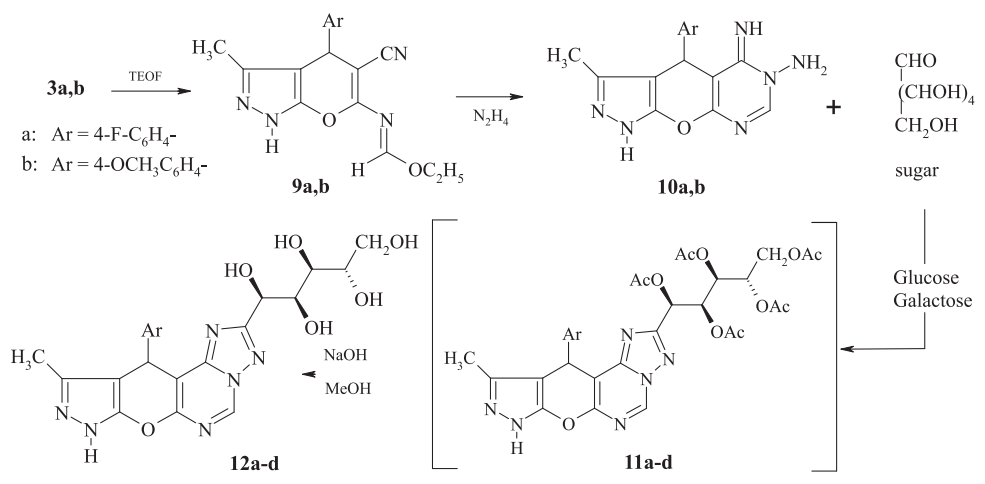

Scheme 4

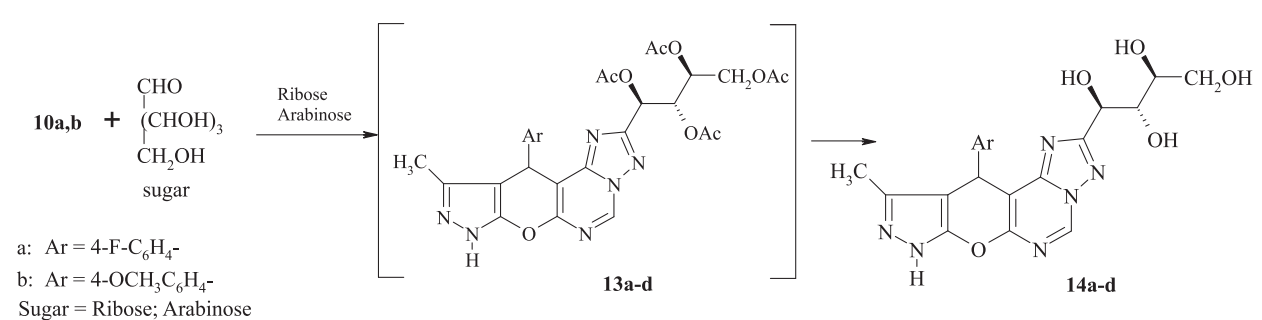

Scheme 5

Deprotection of the acyclic $C$-nucleosides 11a-d and 13a-d could be achieved when they were stirred in the methanolic sodium methoxide solution at room temperature to give good yields of 2-(glycosyl and/or pyranosyl)-11-(4-aryl)-10-methyl-8,11-dihydropyrazolo[4',3':5,6] pyrano[3,2-e][1,2,4] triazolo[1,5-c]pyrimidines $\mathbf{1 2 a - d}$ and $\mathbf{1 4 a - d}$, respectively.

\section{Antimicrobial screening}

Table IV and V show the results of the antimicrobial screening of the new compounds against some Gram-positive, Gram-negative bacteria and fungi.

MIC for compound $\mathbf{6 a}$ is lower than that of ofloxacine against B. subtilis (0.3 vs. 0.4 $\mu \mathrm{mol} \mathrm{L}{ }^{-1}$ ) and equal to that of ofloxacine against S. lutea (both MICs $0.25 \mu \mathrm{mol} \mathrm{L}{ }^{-1}$ ).

Such a high activity may be due to 4-fluorophenyl attached to the pyrazolopyrane and the presence of $\mathrm{N}$-glycoribosyl ring, which increases the antibacterial activity. Compound $\mathbf{6} \mathbf{b}$ was found $\left(M C_{B \text {. subtilis }}=0.5 \mu \mathrm{mol} \mathrm{L} \mathrm{L}^{-1}\right)$ nearly as active as ofloxacine, while compounds

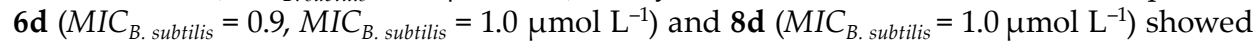
good activities atributable to the presence of the electron withdrawing hydroxyl groups in pyranozyl moeity. Other newly synthesized compounds exhibited slight antibacterial activity, and inactive against fungi compared to the reference drug (miconazole). 


\section{Antitumor activity and structure activity relationship}

The effect of compounds $\mathbf{6 a}, \mathbf{b}, \mathbf{d}, \mathbf{8 a}, \mathbf{d}, \mathbf{1 2} \mathbf{a}$ and $\mathbf{1 4 a}$ was evaluated through in vitro growth of three human tumor cell lines representing three tumor types, namely, liver cancer (HepG2), colon cancer (HT29) and breast adenocarcinoma (MCF-7), after continuous exposure for $48 \mathrm{~h}$. The results are summarized in Table VI. All compounds were able to inhibit the growth of human tumor cell lines in a dose-dependent manner. Compounds $N$-( $\beta$-D-ribofuranosyl)-6\{[(1E)-4-chlorophenyl)methylene]amino\}4-(4-florophenyl)-3-methyl-1,4-dihydropyrano[2,3-c]pyrazole-5-carbonitrile (6a) and $N$-( $\beta$-D-xylofuranosyl)-6 $\{[(1 E)$ 4-chlorophenyl)methylene]amino\}4-(4-florophenyl)-3-methyl-1,4-dihydropyrano[2,3-c] pyrazole-5-carbonitrile (6b) exhibited the highest cytotoxicity effect on the three tumor cell lines, but still lower than that of doxorubicin, one of the most effective anticancer agents which was used as the reference drug. Inhibitions of both compounds may be attributed to the presence of the sugar moiety $N$-( $\beta$-D-ribofuranosyl $), N-(\beta$-D-xylofuranosyl) and 4-florophenyl attached to the pyrane ring. On the other hand, compounds $6 \mathbf{d}, \mathbf{8 a}, \mathbf{d}$, 12a and 14a showed lower growth inhibitory effect. By comparing activities of compounds $\mathbf{6 a}, \mathbf{b}$ and $\mathbf{8 a}, \mathbf{d}$, it is observed that $\mathbf{6 a}, \mathbf{b}$ showed higher cytotoxicity compared to $\mathbf{8 a}, \mathbf{d}$, probably due to the presence of $N$-furanosyl, which increases cytotoxicity compared to $N$-pyranozyl. Moreover, it is convenient to compare the activity of 5-(ribosyl)-[11-(4-florophenyl)10-methyl-8,11-dihydropyrazolo[4',3':5,6] pyrano[3,2-e][1,2,4]triazolo[1,5-c]pyrimidin-2-yl (14a) with that of 5-(glycosyl)-[11-(4-floro-phenyl)-10-methyl-8,11-dihydropyrazolo[4,'3':5,6] pyrano[3,2-e][1,2,4]triazolo[1,5-c]pyrimidin-2-yl (12a). The former compound derived from aldopentoses is more active than when derived from aldohexose.

\section{CONCLUSIONS}

The objective of the present study was to synthesize and investigate the antimicrobial and anticancer activity of some novel $N$ - and $C$-nucleosides of pyranopyrazolo and pyrazolopyranopyrimidine derivatives. Compound $N$-( $\beta$-D-ribofuranosyl)-6\{[(1E)-4-chlorophenyl)-methylene]amino\}4-(4-florophenyl)-3-methyl-1,4-dihydropyrano[2,3-c]pyrazole5 -carbonitrile (6a) was found to exert the most potent antibacterial activity, higher or equal to the activity of ofloxacine. On the other hand, compounds $\mathbf{6} \mathbf{a}, \mathbf{b}$ exhibited promising antitumor activity on the three tumor cell lines.

Acknowledgements. - The authors thank the Micro-analytical Unit, Cairo University, Egypt, for micro-analytical data, IR, NMR and mass spectra. The authors are also grateful to the staff of the Bacteriology Laboratory, National Research Center (Cairo, Egypt) and the staff of the Antitumor Laboratory, at the National Cancer Institute, Cairo University, Egypt.

\section{REFERENCES}

1. E. De Clercq, Antiviral drugs in current clinical use, J. Clin. Virol. 30 (2004) 115-133; DOI: 10.1016/j. jcv.2004.02.009.

2. P. J. O'Dwyer, S. A. King, J. Plowman, C. K. Grieshaber, D. F. Hoth and B. Leyland-Jones, Pyrazole: preclinical reassessment, Invest. New Drug 6 (1988) 305-310. 
3. A. Bekhit and T. Abdel-Aziem, Design, synthesis and biological evaluation of some pyrazole derivatives as anti-inflammatory-antimicrobial agents, Bioorg. Med. Chem. 12 (2004) 1935-1945; DOI: 10.1016/j.bmc.2004.01.037.

4. K. L. Kees, J. J. Fitzgerald, K. E. Steiner, J. F. Mattes, B. Mihan and T. Tosi, New potent antihyperglycemic agents in $\mathrm{db} / \mathrm{db}$ mice: synthesis and structure-activity relationship studies of (4-substituted benzyl) (trifluoromethyl)pyrazoles and -pyrazolones, J. Med. Chem. 39 (1996) 3920-3928.

5. A. Geronikaki, E. Babaev, J. Dearden, W. Dehaen, D. Filimonov, I. Galaeva, V. Krajneva, A. Lagunin, F. macaev, G. Molodavkin, V. Poroikov, S. Pogrebnoi, V. Saloutin, A. Stepanchikova, E. Stingaci, N. Tkach, L. Vlad and T. Voroninaf, Design, synthesis, computational and biological evaluation of new anxyiolytics, Bioorg. Med. Chem. 12 (2004) 6559-6568; DOI: 10.1016/j. bmc.2004.09.016.

6. D. Sil, R. Kumar, A. Sharon, P. R. Malik and V. Ram, Stereoselective alkenylation of a 1,3-disubstituted pyrazol-5-one through ring transformation of 2H-pyran-2-ones, Tetrahedron Lett. 46 (2005) 3807-3809; DOI: 10.1016/j.tetlet.2005.03.207.

7. J. S. Larsen, M. A. Zahran, E. B. Pedersen and C. Nielsen, Synthesis of triazenopyrazole derivatives as potential inhibitors of HIV-1, Monatsh. Chem. 130 (1999) 1167-1173.

8. A. Arrieta, J. R. Carrillo, F. P. Cossl, E. Ortiz, M. J. Gomez-Escalonilla, A. de la Hoz, F. Langa and A. Moreno, Efficient tautomerization hydrazone-azomethine imine under microwave irradiation. Synthesis of [4,3'] and [5,3']bipyrazoles, Tetrahedron 54 (1998) 13167-13180; DOI: 10.1016/S00404020(98)00798-4.

9. G. M. Nitulescu, C. Draghici and O. T. Olaru, New potential antitumor pyrazole derivatives: Synthesis and cytotoxic evaluation, Int. J. Mol. Sci. 14 (2013) 21805-21818; DOI: 10.3390/ijms141121805.

10. J. L. Wang, D. Liu, Z. J. Zhang, S. Shan, X. Han, S. M. Srinivasula, C. M. Croce, E. S. Alnemri and Z. Huang, Structure-based discovery of an organic compound that binds Bcl-2 protein and induces apoptosis of tumor cells, Proc. Natl. Acad. Sci. USA 97 (2000) 7124-7129.

11. E. S. El-Tamany, F. A. El-Shahed and B. H. Mohamed, Synthesis and biological activity of some pyrazole derivatives, J. Serb. Chem. Soc. 64 (1999) 9-18.

12. M. E. A. Zaki, H. S. Soliman, O. A. Hiekal and A. E. Rashad, Pyrazolopyranopyrimidines as a class of anti-inflammatory agents, Z. Naturforsch. C. 61 (2006) 1-5.

13. F. M. Abdelrazek, P. Metz, N. H. Metwally and S. F. El-Mahrouky, Synthesis and molluscicidal activity of new cinnoline and pyrano[2,3-c]pyrazole derivatives, Arch. Pharm. 339 (2006) 456-460.

14. F. M. Abdelrazek, P. Metz, O. Kataeva, A. Jager and S. F. EI-Mahrouky, Synthesis and molluscicidal activity of new chromene and pyrano[2,3-c]pyrazole derivatives, Arch. Pharm. 340 (2007) 543-548.

15. N. Foloppe, L. M. Fisher, R. Howes, A. Potter, A. G. S. Robertson and A. E. Surgenor. Identification of chemically diverse Chk1 inhibitors by receptor-based virtual screening, Bioorg. Med. Chem. 14 (2006) 4792-4802; DOI: 10.1016/j.bmc.2006.03.021.

16. S. A. El-Assiery, G. H. Sayed and A. Fouda, Synthesis of some new annulated pyrazolo-pyrido (or pyrano) pyrimidine, pyrazolopyridine and pyranopyrazole derivatives, Acta Pharm. 54 (2004) 143-150.

17. C. M. Ashraf, S. S. Ali and A. Hussain, Organic reaction in aqueous solution, Part-VIII: Effect of Sonication on the reaction of thiosemicarbazide with ethyl acetoacetate, J. Chem. Soc. Pak. 20 (1998) 145-149.

18. M. M. H. Bhuiyan, M. I. Hossain, M. A. Alam and M. M. Mahmud, Microwave assisted Knoevenagel condensation: synthesis and antimicrobial activites of some arylidene-malononitriles, Chem. J. 2 (2012) 31-37.

19. S. R. Jain and A. Kar, The antibacterial activity of some essential oils and their combinations. Planta Med. 20 (1971) 118-123; DOI: 10.1055/ s-0028-1099675. 
20. H. Ericsson and J. C. Sherris, Antibiotic sensitivity testing. Report of an international collaborative study, Acta Pathol. Microbiol. Scand. Sect. B 217 (1971) 1-90.

21. P. Skehan, R. Storeng, D. Scudiero, A. Monks, J. McMahon, D. Vistica, J. T. Warren, H. Bokesch, S. Kenny and M. R. Boyd, New colorimetric cytotoxicity assay for anticancer drug screening, J. Natl. Cancer Inst. 82 (1990) 1107-1112; DOI: 10. 1093/ jnci /82.13.1107.

22. A. Monks, D. Scudiero, P. Skehan, R. Shoemaker, K. Paul, D. Vistica, C. Hose, J. Langley, P. Cronise, A. Vaigro-Wolff, M. Gray-Goodrich, H. Campbell, J. Mayo and J. M. Boyd, Feasibility of a highflux anticancer drug screen using a diverse panel of cultured human tumor cell lines, J. Natl. Cancer Inst. 83 (1991) 757-776; DOI: 10. 1093/ jnci /83.11.757.

23. A. B. A. El-Gazzar, H. N. Hafez and G. A. M. Nawwar, New acyclic nucleoside analogues as potential analgesic, anti-inflammatory, anti-oxidant and anti-microbial derived from pyrimido[4,5b]quinolines, Eur. J. Med. Chem. 44 (2009) 1427-1436; DOI: 10.1016/j.-ejmech.2008.09.030. 